\title{
Light-front wave functions of mesons, baryons, and pentaquarks with topology-induced local four-quark interaction
}

\author{
Edward Shuryak \\ Department of Physics and Astronomy, Stony Brook University, Stony Brook, New York 11794-3800, USA
}

(Received 7 October 2019; published 11 December 2019)

\begin{abstract}
We calculate light-front wave functions of mesons, baryons and pentaquarks in a model including constituent mass (representing chiral symmetry breaking), harmonic confining potential, and four-quark local interaction of 't Hooft type. The model is a simplified version of that used by Jia and Vary. The method used is numerical diagonalization of the Hamiltonian matrix, with a certain functional basis. We found that the nucleon wave function displays strong diquark correlations, unlike that for the Delta (decuplet) baryon. We also calculate a three-quark-five-quark admixture to baryons and the resulting antiquark sea parton distribution function.
\end{abstract}

DOI: 10.1103/PhysRevD.100.114018

\section{INTRODUCTION}

\section{A. Various roads towards hadronic properties}

Let us start with a general picture, describing various approaches to the theory of hadrons, identifying the following:

(i) Traditional quark models (too many to mention here) are aimed at calculation of static properties (e.g., masses, radii, magnetic moments etc.). Normally all calculations are done in the hadron's rest frame, using certain model Hamiltonians. Typically, chiral symmetry breaking is included via effective "constituent quark" masses; the Coulomb-like and confinement forces are included via some potentials. In some models a "residual" interaction is also included, via some four-quark terms of the Nambu-Jona-Lasinio (NJL) type.

(ii) Numerical calculation of Euclidean-time two- and three-point correlation functions is another general approach, with source and sink operators creating a state with needed quantum numbers, and the third one in between, representing the observable. Originally started from small-distance operator product expansion (OPE) and the QCD sum rule method, it moved to intermediate distances (see review e.g., in [1]), and it is now mostly used at large time separations $\Delta \tau$ (compared to inverse mass gaps in the problem $1 / \Delta M$ ) by the lattice gauge theory (LGT) simulations. This condition ensures the

Published by the American Physical Society under the terms of the Creative Commons Attribution 4.0 International license. Further distribution of this work must maintain attribution to the author(s) and the published article's title, journal citation, and DOI. Funded by SCOAP. "relaxation" of the correlators to the lowest mass hadron in a given channel.

(iii) Light-front quantization using also certain model Hamiltonians, aimed at the set of quantities, available from experiment. Deep inelastic scattering (DIS), as well as many other hard processes, use factorization theorems of perturbative QCD and nonperturbative parton distribution functions (PDFs). Hard exclusive processes (such as e.g., form factors) are described in terms of nonperturbative hadron on-light-front wave functions (LFWFs); for reviews see [2,3].

(iv) A relatively new approach is "holographic QCD," describing hadrons as quantum fields propagating in the "bulk" space with extra dimensions. It was originally supposed to be a dual description to some strong-coupling regime of QCD, and therefore it was mostly used for description of the quark-gluon plasma (QGP) phase at high temperatures. Nevertheless, its versions including confinement (via dilaton background with certain "walls" [4]) and quark-related fields (especially in the so-called Veneziano limit in which both the number of flavors and colors are large $N_{f}, N_{c} \rightarrow \infty, N_{f} / N_{c}=$ fixed [5]) do reproduce hadronic spectroscopy, with nice Regge trajectories. The holographic models also led to an interesting revival of baryons-as-solitons-type models, generalizing skyrmions and including also vector meson clouds. Brodsky and de Teramond [6] proposed to relate the wave functions in extra dimension $z$ to those on the light front, identifying $z$ with a certain combination of the light cone variables $\zeta$. Needless to say, all of these are models constructed from the "bottom up," but with welldefined Lagrangians and some economic set of parameters, from which a lot of (mutually consistent) predictions can be worked out. 
While (i) and (iv) remain basically in the realm of model building, (ii) remains the most fundamental and consistent approach. Lattice studies, starting from the first principles of QCD, have convincingly demonstrated that they correctly include all nonperturbative phenomena. They do display chiral symmetry breaking and confinement, and they reproduce accurately hadronic masses. Yet lattice evaluation of PDFs and light-front wave functions remains difficult. The light-front direction, on the other hand, for decades relied on perturbative QCD, in denial of most of nonperturbative physics.

The aim of the present paper is to bridge the gap between approaches (ii) and (iii). It is a kind of pilot project, using a particular quark model (deliberately stripped down to its "bare bones") and then performing consistent dynamical calculations of the light-front wave functions in it. It follows directly the approach of Jia and Vary [7] to pion and rho mesons. We extended its applications to baryons, the Delta (decuplet) and the proton (the octet), five-quark systems (pentaquarks), and finally to three-quark-fivequark mixing, dynamically addressing the issue of the nonperturbative antiquark sea.

The model Hamiltonian has three terms: (i) the constituent mass term, representing the chiral symmetry breaking, (ii) harmonic-type confining potential, and last but not least (iii) the four-quark local interaction of NJL type. One simplification we use is to consider only the longitudinal degrees of freedom, ignoring the transverse motion. Another is to reduce the complicated NJL operator to a single local topology-induced 't Hooft vertex [8]. This latter step is explained in the next subsection.

\section{B. Topology-induced multiquark interactions}

Nambu and Jona-Lasinio's 1961 paper [9] was an amazing breakthrough. Before the word "quark" was invented, and one learned anything about quark masses, the notions of chiral symmetry and its spontaneously breaking were postulated. They postulated the existence of four-fermion interaction, with some coupling $G$, strong enough to make a superconductorlike gap even in fermionic vacuum. The second important parameter of the model was the momentum cutoff $\Lambda \sim 1 \mathrm{GeV}$, below which their hypothetical attractive four-fermion interaction operates.

After the discovery of QCD, gauge field monopoles and instantons, a very curious relation was found [8], between the Dirac operator and background gauge topology: they have certain zero modes related to the topological charge. This mathematical phenomenon has direct physical consequences: the multiquark interaction vertex described by the so-called 't Hooft effective Lagrangian. Since in QCD it includes all three flavors of light quarks, $u, d, s$, it is a sixquark effective vertex, schematically shown in Fig. 1(a). Note its key feature, opposite chiralities $L, R$ of the incoming and outgoing quarks: it is so because in order to have zero modes of the Dirac equation, quarks and

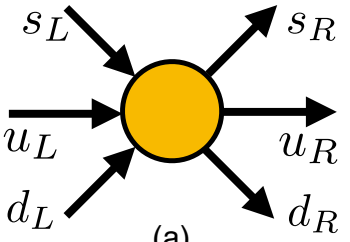

(a)

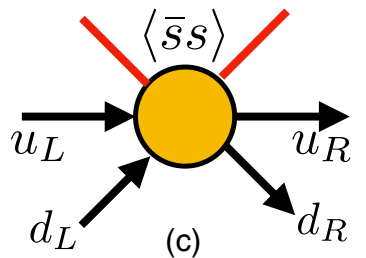

(c)

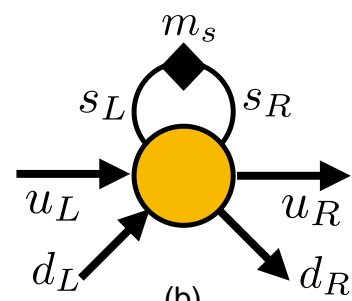

(b)

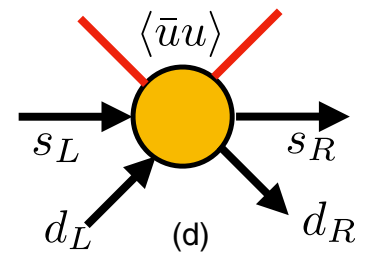

FIG. 1. The schematic form of the six-quark 't Hooft effective Lagrangian is shown in (a). If quarks are massive, one can make a loop as shown in (b), reducing it to a four-fermion operator. Note a black diamond indicating the mass insertion into a propagator. We only show it for the $s$ quark, hinting that for $u, d$ their masses are too small to make such a diagram really relevant. In (c),(d) we show other types of effective four-fermion vertices, appearing because some quark pairs can be absorbed by nonzero quark condensates (red lines).

antiquarks should have the same magnetic moments. Unlike vectorial interaction with nontopological glue, this Lagrangian directly connects the left and right components of quark fields, explicitly breaking $U(1)_{a}$ chiral symmetry.

With the advent of the instanton liquid model (ILM) [10] it became clear that it provides an explanation of the origins of the hypothetical NJL interaction. The NJL coupling $G_{\mathrm{NJL}}$ and cutoff $\Lambda_{\mathrm{NJL}}$ were substituted by two other parameters, the instanton 4D density $n$ and the typical size $\rho$. Like the NJL action, the 't Hooft effective action also preserves the $S U\left(N_{f}\right)$ chiral symmetry, but it is also strong enough to break the symmetry spontaneously, creating nonzero quark condensates $\langle\bar{s} s\rangle,\langle\bar{u} u\rangle \neq 0$ appearing in diagrams (c) and (d) of Fig. 1. The residual four-quark $(\bar{u} u)(\bar{d} d)$ interaction induced by the diagrams (b) and (c) is the one used below. Note that, unlike the NJL action, it explicitly breaks the chiral $U_{c}(1)$ symmetry.

In 1990s the so-called "interacting instanton liquid model," which numerically solved the vacuum properties using the 't Hooft Lagrangian to all orders, provided hadron spectroscopy and Euclidean correlation functions; for a review see [11]. Recent advances to finite temperatures and QCD phase transitions at finite temperature are based on instanton constituents, the "instanton dyons"; we do not go into that here and only comment that the structure of the 't Hooft Lagrangian remains the same.

In this pilot study we would simplify this residual fourfermion interaction, as much as possible, assuming that the 't Hooft effective action is local. This implies that the instanton radius (or $1 / \Lambda_{\mathrm{NJL}}$ ) is much smaller than typical 
hadronic sizes, $\rho \ll R_{\text {hadrons, }}$, and therefore can be neglected. With this assumption, the residual four-fermion interaction has only one parameter, the coupling.

It needs to be stated that such simplification goes with a certain price: the wave functions get singular at $x \rightarrow 1$, causing bad convergence of their expansion in basis functions, so we will terminate series by hand. Let us add that the small size of QCD topological objects also produces technical difficulties for lattice simulations: many quantities (and PDF moments are among them; see e.g., [12]) show significant dependence on the value of lattice spacing $a$ down to very fine lattices, with $a \sim 1 /(2-3 \mathrm{GeV})$, so the continuum extrapolation $a \rightarrow 0$ is a nontrivial step.

The small sizes of instantons and instanton-dyons explain a few other puzzles, known in hadronic physics and by lattice practitioners. We will not discuss phenomena related to strange quark mass in this work, but notice in passing, the "puzzle of strong breaking of the $S U(3)$ flavor symmetry." Naively, in NJL-like models

$$
m_{s} \sim 0.1 \mathrm{GeV} \ll \Lambda_{\mathrm{NJL}} \sim 1 \mathrm{GeV}
$$

is a small parameter, and expansion in it should be well behaved. However, it is far from being seen in the real world and lattice numerical data. One particular manifestation of it, observed e.g., in recent lattice work already mentioned [12], is that PDF moments for various octet baryons $N, \Lambda, \Sigma$ are very different. The $S U(3)$ flavor symmetry is broken at a $100 \%$ level. Its explanation in ILM is also related to small sizes of the topological objects. The four-fermion vertex of Fig. 1(b) is proportional to the (chirality-flipping) factor $m_{s} \rho$. (The appearance of $\rho$ is obvious from dimensional considerations.) The effective vertices generated by diagrams (c) and (d) are proportional to the respective quark condensates, as indicated. For convenience, the sum of (b) and say the (c) vertex is written in a form $\left(m_{s}+M_{s}^{*}\right) \rho$ where the so-called "determinantal mass" $M^{*}$ (not to be confused with the constituent quark mass) can be calculated for small-size instanton á la OPE to be

$$
M_{f}^{*}=\frac{2 \pi^{2}}{3}\left|\left\langle\bar{q}_{f} q_{f}\right\rangle\right| \rho^{2}
$$

and, by coincidence of numbers, is comparable to $m_{s}$. Therefore, the effective four-fermion interactions for light and strange quarks turn out to be a factor of 2 different.

Although we will not discuss chiral symmetry breaking and chiral perturbation theory below, it is tempting to mention one more observation that is very puzzling to lattice practitioners. It is not related to the strange quark mass, but to the light quark masses $m_{u}, m_{d}$. Those are only a few $\mathrm{MeV}$ in magnitude and, naively, a chiral extrapolation to $m_{u}, m_{d} \rightarrow 0$ should just be linear. Yet in practice, many observables show nonlinear mass dependencies. The answer, at least the one coming from ILM, is that the chiral condensate, made of collectivized instanton zero modes, has a spread of Dirac eigenvalues proportional to overlaps of zero modes of individual instantons. To form a condensate, the quark needs to hop from one instanton to the next. The overlap of their zero modes is surprisingly small because the topology ensemble is rather dilute:

$$
\Delta \lambda \sim \rho^{2} / R^{3} \sim 20 \mathrm{MeV}
$$

comparable to quark masses used on the lattice. Here $R \sim 1 \mathrm{fm}$ is a typical distance between instantons. It is $1 / R^{3}$ because such is the propagator of a massless quark in $4 \mathrm{D}$, and two $\rho$ factors are two couplings of a quark to two instantons.

\section{Comments on perturbative evolution and twist expansion}

Completing the Introduction, we would like to add some comments clarifying relations between the model calculation of the light-front wave functions (LFWFs), presented below and in other works, with studies of the perturbative processes, involving gluon and quark-antiquark production. While generic by themselves, these comments have prompted discussion in the literature, so perhaps they are worth repeating.

The hadrons - e.g., a pion or a fully polarized protonare of course single quantum states, described by their multibody wave functions. On the other hand, the PDFs we used to deal with using the parton model are single-body density matrices. In principle, they should be calculated from the wave functions, by integrating out variables relating to other partons. A single struck parton, being a subsystem, possesses entanglement entropy with the rest of the state, as recently pointed out by Kharzeev and Levin [13].

Furthermore, the celebrated and widely used DokshitzerGribov-Lipatov-Altarelli-Parizi (DGLAP) evolution equation is basically a version of the Boltzmann kinetic equation, with "splitting functions" describing the gain and loss in PDFs as the resolution $Q^{2}$ changes. As for any Boltzmann equation, one can define the (ever increasing) entropy. It is also important to note, as any other singlebody Boltzmann equation, that DGLAP is based on the implicit assumption that higher correlations between bodies are small and can be neglected. Therefore, it may appear strange that DGLAP is successfully applied to the nucleon, in which diquark correlations are known to be so strong, that it often is treated as a two-body system (e.g., the nucleon Regge trajectories are nearly the same as for mesons).

To understand that there is no contradiction, one needs to have a look at evolution, including not only the leading twist operators, whose $\mathrm{pQCD}$ evolution is described by DGLAP, but the higher twist operators as 
well. In particular, the general OPE expansion for deepinelastic scattering includes gluon-quark and even fourfermion operators; see e.g., [14]. Their matrix elements are precisely the place where interparton correlations are included. However, these operators are suppressed by powers of $1 / Q^{2}$ and are normally ignored in DGLAP applications for hard processes at large $Q$.

The three lessons from this discussion are as follows. (i) A nucleon has no entropy, but its PDFs have it. (ii) DGLAP is a kinetic equation, ignoring correlations between partons but explaining the entropy growth. It is good at high $Q \gg 1 \mathrm{GeV}$. (iii) Attempting to build a bridge between hadronic models and pQCD description, one thinks about the scale $Q \sim 1 \mathrm{GeV}^{2}$, where correlations and higher twist effects need to be included. Observing parton correlations is hard, and not done yet, but still in principle possible.

\section{FEW-BODY KINEMATICAL VARIABLES ON THE LIGHT FRONT}

A textbook introduction to few-body quantum mechanics starts usually with an introduction of Jacobi coordinates, eliminating the center-of-mass motion. Those can be used for transverse coordinates, but not for momentum fractions $x_{i}, i=1 \ldots N$. In this section we propose a set of $N-1$ coordinates in which one can conveniently describe dynamics with longitudinal momentum fractions $x_{1} \ldots x_{N}$, with the constraint

$$
\sum_{i}^{N} x_{i}=1
$$

But before we do so, let us comment that the number of particles $N$ and variables $N-1$ in the problems to be dealt with need not be fixed. Although such a situation is unlike what is usually described in textbooks, it is rather common in realistic applications of quantum mechanics. For example, complicated atoms or nuclei are described in a basis with closed shells, plus several nucleons (or electrons) above it, plus an indefinite number of particle-hole pairs. Hadronic wave functions similarly should have an indefinite number of quark-antiquark pairs.

In general, LFWFs consist of sectors with $N$ particles, with $N$ changing from some minimal number $N_{\min }$ (2 for mesons, 3 for baryons) to infinity. The variables should satisfy momentum normalization in Eq. (3).

In this paper we will make some drastic simplifications. In particular,

(i) We will ignore transverse momenta, as already mentioned, and focus on the LFWF dependence on the longitudinal momentum fractions $x_{i}$ only.

(ii) As we focus on four-quark interactions, we will ignore gluons and processes involving them. So our particles will be only quarks. They would be dressed due to chiral symmetry breaking and thus have effective "constituent quark masses."

(iii) While each $N$-sector will be described by a space with a certain appropriate number of basis states, the corresponding polynomials, we will truncate the Hilbert space to minimal and next-to-minimal $\left(N_{\min }\right.$ and $N_{\min }+2$ ) sectors. While the former dominate the valence quark structure functions, the latter will be needed to discuss the "sea" quarks and antiquarks.

So, our operational Hilbert space would include sectors with two and four quarks for mesons, and three and five for baryons. Let us discuss these sectors subsequently, explaining more convenient variables and the integration measure for each sector. In selecting the kinematical variables one would like to include the constraint explicitly, yet keeping the integration measure factorizable and within the boundaries.

The two-particle sector is the simplest case. With two momentum fractions, $x_{1}, x_{2}$ and the constraint (3) there remains a single variable. For reasons to become clear soon, we select it to be their difference:

$$
s=x_{1}-x_{2}
$$

in terms of which

$$
x_{1}=\frac{1+s}{2}, \quad x_{2}=\frac{1-s}{2} .
$$

The commonly used integration measure takes the form

$$
\int d x_{1} d x_{2} \delta\left(x_{1}+x_{2}-1\right) x_{1} x_{2} \ldots=\int_{-1}^{1} d s \frac{(1-s)(1+s)}{4} \ldots
$$

The polynomials used in this case, with natural weight $(1-s)^{2}$, are Gegenbauer polynomials $C_{n}^{3 / 2}(s)$ or Jacobi $P_{n}^{1,1}(s)$.

The three-particle sector has been discussed extensively in the literature such as [15]. Two kinematical variables suggested in this case are

$$
s=\frac{x_{1}-x_{2}}{x_{1}+x_{2}}, \quad t=x_{1}+x_{2}-x_{3}
$$

in terms of which

$$
x_{1}=\frac{(1+s)}{2} \frac{(1+t)}{2}, \quad x_{2}=\frac{(1-s)}{2} \frac{(1+t)}{2},
$$

and the integration measure 


$$
\begin{aligned}
\int & \left(\prod_{i} d x_{i}\right) \delta\left(\sum_{i} x_{i}-1\right)\left(\prod_{i} x_{i}\right) \ldots \\
= & \int_{-1}^{1} \int_{-1}^{1} d s d t \frac{(1-s)(1+s)(1-t)(1+t)^{3}}{2^{5}} \ldots
\end{aligned}
$$

Note that it is indeed factorized. Therefore one can split it into two and select an appropriate functional basis. In its choice we however would deviate from that in [15] (because we do not consider perturbative renormalization and anomalous dimensions of the operators) and will use

$$
\psi_{n, l}(s, t) \sim P_{n}^{1,1}(s) P_{l}^{1,3}(t)
$$

The four-particle sector follows the previous example. Three variables are defined as

$$
\begin{aligned}
& s=\frac{x_{1}-x_{2}}{x_{1}+x_{2}}, \quad t=\frac{x_{1}+x_{2}-x_{3}}{x_{1}+x_{2}+x_{3}}, \\
& u=x_{1}+x_{2}+x_{3}-x_{4},
\end{aligned}
$$

producing the integration measure

$$
\begin{aligned}
\int & \left(\prod_{i} d x_{i}\right) \delta\left(\sum_{i} x_{i}-1\right)\left(\prod_{i} x_{i}\right) \ldots \\
= & \int_{-1}^{1} \int_{-1}^{1} \int_{-1}^{1} d s d t d u \frac{(1-s)(1+s)(1-t)(1+t)^{3}(1-u u)(1+u u)^{5}}{32768} \ldots
\end{aligned}
$$

The functional basis is then

$$
\psi_{n, l, m}(s, t) \sim P_{n}^{1,1}(s) P_{l}^{1,3}(t) P_{m}^{1,5}(u) .
$$

The five-particle sector is our last example. Here the four variables, collectively called $z_{i}, i=1,2,3,4$, are defined by

$s=\frac{x_{1}-x_{2}}{x_{1}+x_{2}}, \quad t=\frac{x_{1}+x_{2}-x_{3}}{x_{1}+x_{2}+x_{3}}$,

$u=\frac{x_{1}+x_{2}+x_{3}-x_{4}}{x_{1}+x_{2}+x_{3}+x_{4}}, \quad w=x_{1}+x_{2}+x_{3}+x_{4}-x_{5}$.

The principle idea can also be seen from the inverse relations

$$
\begin{aligned}
& x_{1}=\frac{1}{2^{4}}(1+s)(1+t)(1+u)(1+w) \\
& x_{2}=\frac{1}{2^{4}}(1-s)(1+t)(1+u)(1+w) \\
& x_{3}=\frac{1}{2^{3}}(1-t)(1+u)(1+w), \\
& x_{4}=\frac{1}{2^{2}}(1-u)(1+w) \\
& x_{5}=1-x_{1}-x_{2}-x_{3}-x_{4}=\frac{1}{2}(1-w) .
\end{aligned}
$$

The integration measure follows the previous trend, being factorizable:

$$
\begin{aligned}
\int \frac{d s d t d u d w}{16777216}(1-s)(1+s)(1-t)(1+t)^{3} \\
\times(1-u)(1+u)^{5}(1-w)(1+w)^{7} \ldots
\end{aligned}
$$

The orthonormal polynomial basis to be used is by now obvious,

$$
\psi_{l m n k}(s, t, u, w) \sim P_{l}^{1,1}(s) P_{m}^{1,3}(t) P_{n}^{1,5}(u) P_{k}^{1,7}(w)
$$

with normalization constants determined numerically.

\section{MESONS AS TWO-QUARK STATES}

LFWFs for pion and rho mesons were studied by Jia and Vary (JV) [7]. Their Hamiltonian has four terms including (i) the effective quark masses coming from chiral symmetry breaking; (ii) the longitudinal confinement; (iii) the transverse motion and confinement; and, last but not least, (iv) the NJL four-quark effective interaction

$$
\begin{aligned}
H & =H_{M}+H_{\mathrm{conf}}^{\|}+H_{\mathrm{conf}}^{\perp}+H_{\mathrm{NJL}} \\
H_{M} & =\frac{M^{2}}{x_{1}}+\frac{\bar{M}^{2}}{x_{2}} \\
H_{\mathrm{conf}}^{\|} & =\frac{\kappa^{4}}{(M+\bar{M})^{2}} \frac{1}{J(x)} \partial_{x} J(x) \partial_{x} \\
H_{\perp} & =\vec{k}_{\perp}^{2}\left(\frac{1}{x_{1}}+\frac{1}{x_{2}}\right)+\kappa^{4} x_{1} x_{2} \vec{r}_{\perp}^{2}
\end{aligned}
$$

where $M, \bar{M}$ are the masses of the quark and antiquark; $\kappa$ is the confining parameter; $J(x)=x_{1} x_{2}=(1-s)^{2} / 4$ is the integration measure; and $\vec{k}_{\perp}, \vec{r}_{\perp}$ are the transverse 
momentum and coordinate, respectively. If the masses are the same, however, one can simplify it as

$$
\frac{1}{x_{1}}+\frac{1}{x_{2}}=\frac{1}{x_{1} x_{2}}=\frac{4}{\left(1-s^{2}\right)}
$$

and therefore the matrix elements of $H_{M}$ simply lack the factor $\left(1-s^{2}\right)$ normally present in the integration measure.

While we follow the JV work in spirit, we do not follow it in all technical details. The reason is because we intend to widen the scope of the study to three-, four, and five-quark sectors, and we need to keep the uniformity of approach for all of them.

The main deviation is that we will ignore details of the transverse motion, and simply add the mean $\left\langle H_{\perp}\right\rangle$ to hadronic masses, where appropriate. The reason for it is clear: one needs to keep the size of the Hilbert space manageable.

Another modification is technical. The functional basis selected by JV was the eigenfunctions of the two first terms in the Hamiltonian. We do not find it either important or especially beneficial, since the full Hamiltonian is nondiagonal and is numerically diagonalized anyway.

Another provision, limited to two-quark systems, is parity of the wave function, which is a good quantum number. So we select (in this section only) the set of even harmonics (polynomials of $z^{2}$ ).

The set of functions is normalized, so that

$$
\int d s J(s) \psi_{n_{1}}(s) \psi_{n_{2}}(s)=\delta_{n_{1} n_{2}}
$$

and then we calculate the $\left\langle n_{1}|H| n_{2}\right\rangle$ matrix for all three terms of the Hamiltonian. In general, quarks have different masses (as in $K, K^{*}$ mesons). The confining term we borrowed from [7] and use it in the form

$$
H_{\mathrm{conf}}=-\frac{\kappa^{4}}{M_{q}^{2}} \frac{1}{J} \frac{\partial}{\partial s} J(s) \frac{\partial}{\partial s}
$$

where $J(s)=\left(1-s^{2}\right) / 4$ is the integration measure. Two derivatives over momenta are the quantum-mechanical version of the (longitudinal) coordinate squared, so it can be called "a longitudinal version of the harmonic confinement." The measure appearing between the derivatives is there to ensure that this term is a Hermitian operator. The factor 4 is absorbed by changing derivatives over $x$ to those over $s$.

The quartic interaction that we use, as explained already, is not some version of the NJL operator, but rather its significant part, namely the topology-induced 't Hooft Lagrangian. As explained above, its chiral structure is such that it does not contribute to vector mesons (chiral structure $L L+R R)$. It means that by diagonalizing the first two

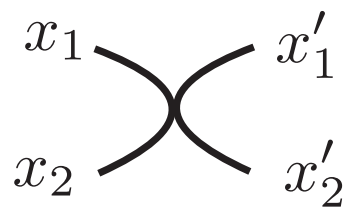

FIG. 2. Kinematical variables in matrix element of four-quark part of the Hamiltonian.

terms of the Hamiltonian we already get predictions for their wave function.

The values of the parameters we use, $M_{q}=0.337 \mathrm{GeV}$, $\kappa=0.227 \mathrm{GeV}$, are just borrowed from the JV paper [7]; one gets the ground state mass $m_{\rho} \approx 700 \mathrm{MeV}$. It is $10 \%$ lower than the experimental and Jia-Vary value, as we neglected the confinement in the transverse direction.

For the ground $\rho$ state we have then the LFWF of the approximate polynomial form given in the Appendix A, as Eq. (A1). Note that it has a rather narrow peak near the symmetry point $s=0$ or $x_{1}=x_{2}$, and it is very small near the kinematical boundaries.

Now we add the four-fermion interaction, shown in Fig. 2. Note that in local approximation the left-hand side and the right-hand side include independent integrations, over $\left(x_{1}, x_{2}\right)$ in one state and over $\left(x_{1}^{\prime}, x_{2}^{\prime}\right)$ in the other. But since this gives the total probability of any values, the corresponding matrix is proportional to this curious matrix $M_{\text {all ones }}$ which has all matrix elements being just 1:

$$
\begin{aligned}
H_{\mathrm{NJL}}^{n_{1}, n_{2}} & = \pm G^{2} M_{\text {all ones }} \\
M_{\text {all ones }}^{n_{1}, n_{2}} & \equiv 1 .
\end{aligned}
$$

The minus sign corresponds to the four-fermion attraction in the pions, and the plus sign to repulsion in the $\eta^{\prime}$ channel. Let us explain why it is so, using the simplest two-flavor case, in which

$$
\left|\pi^{0}\right\rangle \sim \frac{1}{\sqrt{2}}(\bar{u} u+\bar{d} d)
$$

and the isospin zero configuration orthogonal to it $\eta$ (becoming $\eta^{\prime}$ in three-flavor theory) is

$$
|\eta\rangle \sim \frac{1}{\sqrt{2}}(\bar{u} u-\bar{d} d) .
$$

In the matrix elements of the Hamiltonian, $\left\langle\pi^{0}|H| \pi^{0}\right\rangle$ and $\langle\eta|H| \eta\rangle$, the nondiagonal operators $(\bar{u} u)(\bar{d} d)$ contribute with the opposite sign, making the pion light and $\eta$ heavy. The opposite sign here is a well-known manifestation of explicit $U_{a}(1)$ breaking.

Adding this last term of the Hamiltonian to the first two, and diagonalizing, we obtain masses of these states. The coupling we select from the requirement that pions be massless, which leads to mesonic effective coupling $G \approx 0.65 \mathrm{GeV}^{2}$. 

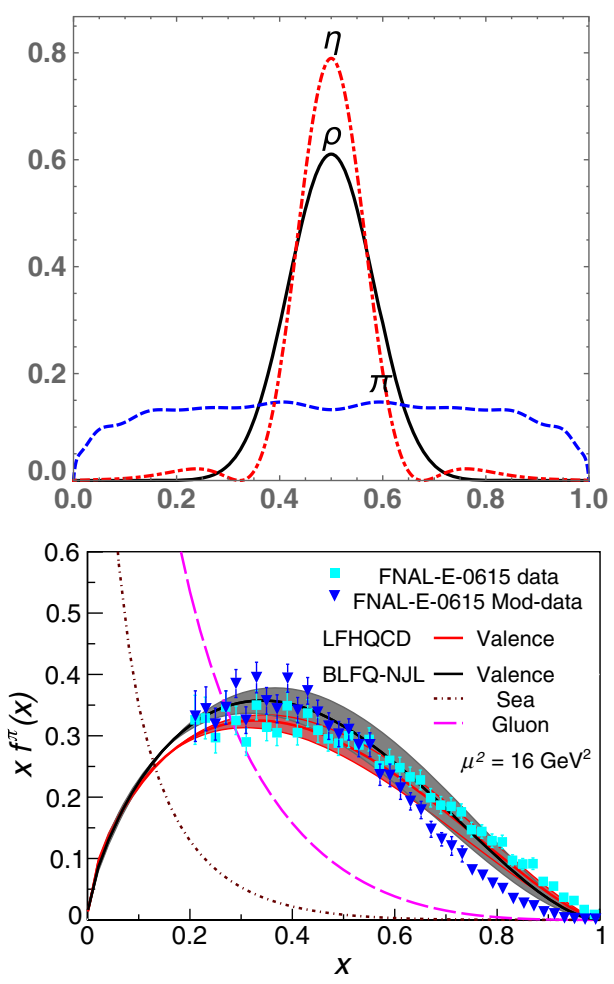

FIG. 3. Upper: Momentum distribution for pion, rho and etaprime mesons, calculated in the model. Lower (from [16]): Comparison between the measured pion PDF (points) and the JV model (lines).

The probability to find a quark at momentum fraction $x$, $P=x(1-x) \psi^{2}(x)$, should correspond to valence structure functions. (Of course, we still are in the two-quark sector and have no sea. There is no pQCD evolution or gluons.)

The results from the diagonalization of $H$, for the lowest states in the rho, pion and eta-prime channels, are plotted in Fig. 3. Note that the predicted PDF for the $\rho$ meson (in which the four-fermion interaction is presumed to be absent) is peaked near the symmetric point $x=1 / 2$, $s=0$. The pion one, in contrast, has a completely different flat shape. As one compares the lower plot to our result, one should keep in mind the fact that the PDF includes also a contribution from sectors with the quark number larger than 2, while ours (so far) do not.

Two historic remarks are in order. (i) One needs to mention an early model of the so-called "double-hump" pion wave function suggested in the 1980s by Chernyak and Zhitnitsky (CZ). In our notations their wave function corresponds to the first harmonics only, $\psi_{\pi}(s) \sim s$. Our result does not of course coincide with it, yet we do find that local four-quark interaction does indeed make the $x$ distribution much flatter [compared to the "asymptotic" distribution given by the measure alone $\sim x(1-x)=$ $\left.\left(1-s^{2}\right) / 4\right]$. (ii) The pion wave function was calculated within the instanton liquid model in [17]: to the extent we can compare them, the results are similar.
Not surprisingly, the PDF for the "repulsive" $\eta^{\prime}$ channel moves in the opposite direction. In fact two maxima are predicted, the larger one above $x=1 / 2$ and a small one near $x=1 / 4$. While these predictions are not possible to verify experimentally, one in principle should be able to compare them with lattice data and other models.

Concluding this section, let us make a few comments on the main simplification, as compared to [7], namely on ignoring wave function dependence on transverse momenta. Note that even in this paper a comparison between wave functions with various cutoffs in the $L$ ( $x$-related) quantum number is studied, with $L_{\max }=8$, 16, 32, while no similar discussion of dependence on ( $\vec{p}_{\perp}$-related) $M_{\max }$ is given. Since the aim of this paper is to extend application of the model up to 5-bodies, doubling of the number of degrees of freedom would obviously expand the functional basis by several orders of magnitude and make calculation impractical. For example, if we would also adopt maximal orbital momentum to be (in their notations) $M_{\max }=2$, as they did, it will bring in $5+3+1=9$ new amplitudes per particle, or a $9^{4}$ larger basis in pentaquarks.

Still, the dependence on $\vec{p}_{\perp}$ remains an open issue. The point we want to make is that it is not just a technical complication with a larger functional basis, but a serious physics issue. Using nonrelativistic language for vector mesons, one would say that they have total angular momentum $J=1$ and possible orbital ones 0 mixed with 2 (reminding one in this respect of the classic nuclear physics system, the deuteron). The admixture of the quadrupole, and the corresponding quadrupole moment, depends on the magnitude of the relativistic effects $\sim v^{2} / c^{2}$ (not too large in this model) as well as the nonlocality of the four-quark interaction. Both in [7] and in this work this interaction is treated as local, ignoring finite sizes of instantons and the related form factors. Therefore we do not think that the model, in its current form, can describe the dependence on $\vec{p}_{\perp}$ correctly.

\section{BARYONS AS THE THREE INTERACTING QUARKS}

In this pilot study we will pay little attention to quark/ antiquark spin states. Like transverse motion, it will be delegated to later studies with a larger functional basis. Let us just mention that the three-quark wave functions we will be discussing are for the delta baryon

$$
\left|\Delta^{++}\right\rangle \sim \psi_{\Delta}\left(x_{i}\right)\left|u^{\uparrow}\left(x_{1}\right) u^{\uparrow}\left(x_{2}\right) u^{\uparrow}\left(x_{3}\right)\right\rangle
$$

and for the proton

$$
\begin{gathered}
|p \uparrow\rangle \sim \psi_{p}\left(x_{i}\right)\left(\left|u^{\uparrow}\left(x_{1}\right) u^{\downarrow}\left(x_{2}\right) d^{\uparrow}\left(x_{3}\right)\right\rangle\right. \\
\left.-\left|u^{\uparrow}\left(x_{1}\right) d^{\downarrow}\left(x_{2}\right) u^{\uparrow}\left(x_{3}\right)\right\rangle\right)
\end{gathered}
$$


and in what follows we will focus on the former component, in which the $d$ quark has the last momentum fraction $x_{3}$. For a review see [18].

Let us just mention that some empirical information about the three-quark LFWF of the nucleons comes from exclusive processes. The theory of these functions is, to our knowledge, reduced to evaluation of certain moments, originally via QCD sum rules and now from lattice studies. The calculation of the pQCD evolution as a function of $Q^{2}$, using the matrix of anomalous dimensions for leading twist operators, was done in [15]. For doing so, it was important to use the so-called conformal basis

$$
\Psi_{N, n}\left(x_{i}\right) \sim(1+t)^{n} P_{N-n}^{(2 n+3,1)}(-t) C_{n}^{3 / 2}(s)
$$

where $P_{l}^{(a, b)}(z)$ and $C_{n}^{m}(z)$ are Jacobi and Gegenbauer polynomials. [Both are defined on $z \in[-1,1]$ and form the orthonormal basis, with the weight functions $(1-z)^{a}$ $(1+z)^{b}$ and $\left(1-z^{2}\right)^{m-1 / 2}$, respectively.] For consistency, we will be using a slightly different set of functions defined (24) as the orthogonal set. (We omit the discussion of normalization coefficients of these functions, which are known but not particularly instructive.)

The first physical effect we would like to incorporate is the part of the effective Hamiltonian coming from chiral symmetry breaking, the constituent quark masses term

$$
H_{\text {mass }}=M_{q}^{2}\left(\frac{1}{x_{1}}+\frac{1}{x_{2}}+\frac{1}{x_{3}}\right) .
$$

The masses we use are the same as those used in the fit for mesons in [7].

The second included effect is confinement. We recall that since $x_{i}$ are momenta, the longitudinal coordinates are quantum conjugate to them, or $i \partial / \partial x_{i}$. Making it as simple as possible, we follow what is done for mesons in [7], so we define this part of the Hamiltonian in the following form, in $s, t$ coordinates:

$H_{\mathrm{conf}}=-\frac{\kappa^{4}}{J(s, t) M_{q}^{2}}\left[\frac{\partial}{\partial s} J(s, t) \frac{\partial}{\partial s}+\frac{\partial}{\partial t} J(s, t) \frac{\partial}{\partial t}\right]$

with the measure function $J(s, t)$ appearing in the $s, t$ integration. Note that the coefficient 4 in the denominator is missing: this is canceled by the factor 4 coming from a difference between derivatives in the $x$ and $s, t$ variables.

The third (and the last) effect we incorporate in this work is the topology-induced four-quark interactions. Note that the topological 't Hooft Lagrangian is flavor antisymmetric. This means that it does not operate e.g., in baryons made of the same flavor quarks, like the $\Delta^{++}=u u u$. Another reason why the 't Hooft vertex should be absent is in any states in which all spin orientations of quarks are the same. For both these reasons, $\Delta^{++}$is not affected by topology effects, therefore serving as a benchmark (like the $\rho$ meson did in the previous sections).

Thus, we have a prediction for the masses of $\Delta$ baryons and their excited states, as well as the corresponding LFWFs. Using the quark mass and the same confining parameter as for the mesons, we get the mass squared of the lowest three-quark state to be $1.28 \mathrm{GeV}^{2}$. Following what we learned from the mesonic case about transverse energy correction, we add it twice and get

$$
M_{\Delta}=\sqrt{1.28+2 \times .12}=1.23 \mathrm{GeV}
$$

in perfect agreement with the experimental Bright-Wigner mass $M_{\Delta}=1.230-1.234 \mathrm{GeV}$.

A couple of other comments are necessary at this point:

(i) Without the confining term, the mass squared of the lowest three-quark state is only slightly smaller, $1.11 \mathrm{GeV}^{2}$. This is because this state is dominated by the lowest harmonics, as we will show below, and the Laplacian in it is zero. In other words, the predicted LFWS of delta is rather flat.

(ii) The mass squared of the next state is $1.679 \mathrm{GeV}^{2}$, and with a transverse correction it leads to

$$
M_{\Delta}=\sqrt{1.679+2 \times 0.12}=1.39 \mathrm{GeV} .
$$

Experimentally, the second $\Delta^{\prime}$ state has a higher Bright-Wigner mass, at $1.5-1.7 \mathrm{GeV}$. We generally do not expect the model to adequately describe the excited states, since transverse degrees of freedom are not really represented in the WF.

The next step is implementation of the four-quark residual interaction. In the notations used (23), we focus on the former proton state, in which the $d$ quark has momentum fraction $x_{3}$. Unlike general NJL operators, the topology-induced one is flavor antisymmetric, acting in the $(u d)$ channel only. So, in principle there can be diagrams

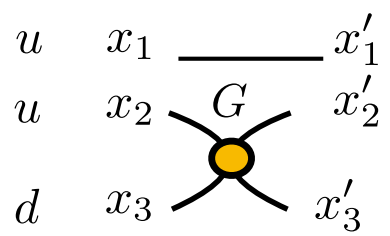

(a)

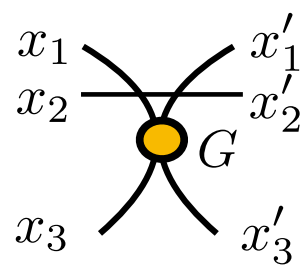

(b)

FIG. 4. Two terms in the Hamiltonian, describing rescattering in the $u\left(x_{2}\right) d\left(x_{3}\right)$ channel (a) and $u\left(x_{1}\right) d\left(x_{3}\right)$ channel (b) due to residual local four-quark interaction. 
(a) and (b), shown in Fig. 4, with interaction in the $(2,3)$ and (1-3) pairs, but none in the $u$ pair due to the Pauli principle for the quark zero modes. Furthermore, the topologyinduced NJL-like local attractive term only appears in the spin-zero diquark channel, and therefore the spin directions of $u$ and $d$ quarks must be opposite. This eliminates the diagram (b). Note also that two $u$ quarks are distinguishable as they have opposite spins: thus there are no exchange diagrams.

The kinematics of the diagram is as follows. In general, a transition amplitude from the initial set $x_{1}, x_{2}, x_{3}$ of kinematical variables to the final ones $x_{1}^{\prime}, x_{2}^{\prime}, x_{3}^{\prime}$ includes four integrations. However, since one of the quarks does not participate, there is always one delta function for the noninteracting quark [e.g., for (a) interacting, $\left.x_{1}=x_{1}^{\prime}\right]$, and the matrix elements of the (a) and (b) diagrams include effectively 3D integrals. Yet since all the functions and kinematical boundaries are already defined in terms of $s, t, s^{\prime}, t^{\prime}$, we preferred to keep full 4D integrations, implementing the delta function approximately, by a narrow Gaussian.

The Hamiltonian matrix for residual interaction we write in the form, for diagram (a),

$$
\begin{aligned}
\left\langle i_{1}\left|H_{a}\right| i_{2}\right\rangle= & -G \int d s d t J(s, t) \psi_{i_{1}}(s, t)^{2} \\
& \times \int d s^{\prime} d t^{\prime} J\left(s^{\prime}, t^{\prime}\right) \psi_{i_{2}}\left(s^{\prime}, t^{\prime}\right)^{2} \delta\left(x_{1}(s, t)\right. \\
& \left.-x_{1}^{\prime}\left(s^{\prime}, t^{\prime}\right)\right) .
\end{aligned}
$$

The minus sign corresponds to attraction in the $u d$ channel. As $G$ increases, the mass of the lowest state decreases: we stop when it becomes equal to the nucleon mass. This allows us to fix our first model parameter

$$
G \approx 17 \mathrm{GeV}^{2} .
$$

(We already noticed in the pion case, that an attractive four-fermion interaction in principle makes the vacuum unstable and creates the massless pion. At the chosen $G$ there are signs of this phenomenon, as imaginary parts of squared masses of higher nucleon states. The nucleon's mass remains real and far from zero.)

Now we are ready to explore properties of the obtained nucleon wave function, which one can read off from the eigenvector of the Hamiltonian.

The simplest quantity to display are single-quark densities, corresponding to nucleon PDFs. In particular, the distribution over $x_{3}$, or the $d$ quark, depends in our notations only on the variable $t$. So, performing integration over the variable $s$ while keeping $t$ arbitrary yields the $d$-quark distribution

$$
d\left(x_{3}=1-2 t\right)=\int_{-1}^{1} d s J(s, t) \Psi_{N}^{2}(s, t) .
$$
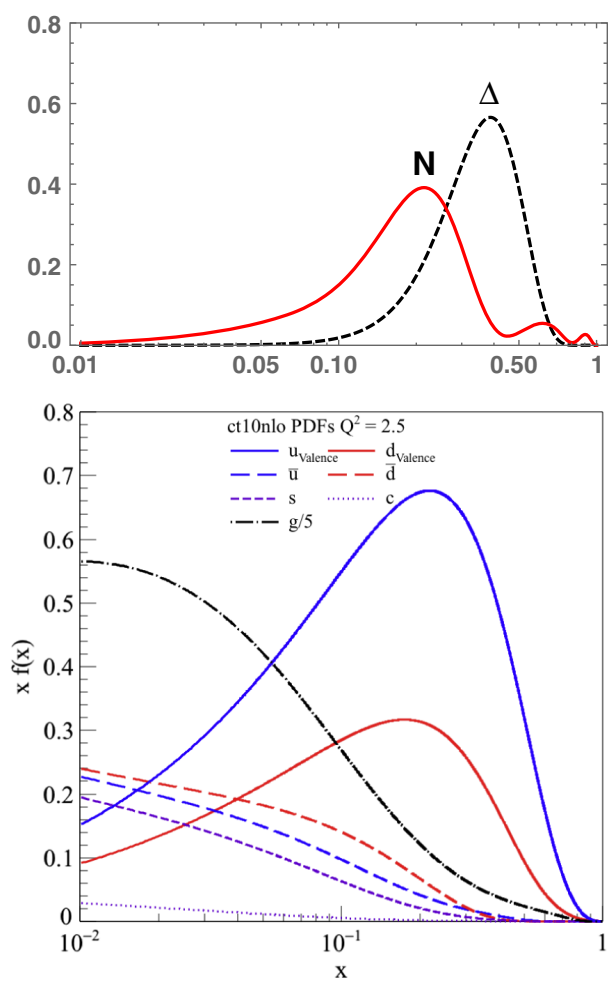

FIG. 5. Upper: Our calculation of the $d$ quark distribution in the nucleon times $x, x d(x)$ (red, solid) and delta (black, dashed) states. For comparison, the lower plot shows empirical structure functions (copied from [16]), where the valence $x d(x)$ is also shown in red.

This distribution multiplied by $x$ is shown in Fig. 5 (upper), for the delta and nucleon wave functions that we obtained. Two comments are in order: (i) the peak in the nucleon distribution moves to lower $x_{d}$, as compared to $x=1 / 3$ expected in $\Delta$ and noninteracting three quarks; (ii) there appears a larger tail toward small $x_{d}$ in the nucleon, but also some peaks at large $x_{d}$. Both are unmistakably the result of local $u d$ pairing (strong rescattering) in a diquark cluster.

Figure 5 (lower), shown for comparison, includes the empirical valence $x d(x)$ distribution, also shown by a red solid line. The location of the peak roughly corresponds to data. The presence of the small- $x_{d}$ tail is also well seen (recall that what is plotted is the distribution times $x$ ). The experimental distribution is of course much smoother than ours. It is an expected feature: our wave function is expected to be "below the pQCD effects," at a resolution of say $Q^{2} \sim 1 \mathrm{GeV}^{2}$, while the lower plot is at $Q^{2}=2.5 \mathrm{GeV}^{2}$, and it includes a certain amount of $\mathrm{pQCD}$ gluon radiation. It also contains higher order correlations between quarks.

Our distribution displays a certain structure, related to the quark-diquark component of the wave function, which may or may not be true. It is definitely present in the model used. As for the data, it is not so obvious: collaborations fitting the data imply simple functional forms, so their smoothness of the empirical PDFs also may be questioned. 


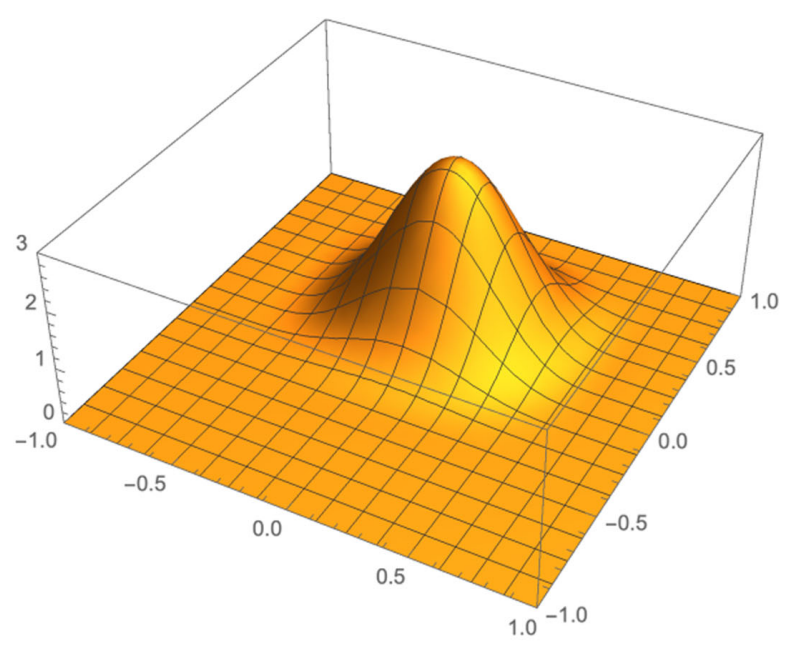

and the model discussed in [19] (see the Appendix). While the delta shows a peak near the symmetry point $x_{1}=x_{2}=$ $x_{3}$ as expected, without any other structures, our nucleon WFs indicate more complicated dynamics. Indeed, there appear several bumps, most prominent near $s \approx 1, t \approx 1$ which is $x_{1} \approx 1$. Apparently, it is about the same peak location which $\mathrm{CZ}$ wave functions wanted to emulate. Such strong peaking corresponds to a large momentum transfer inside the $u d$ diquark clusters. Yet there is also the peak in the middle, roughly corresponding to that in delta. So, the nucleon wave function is a certain coherent mixture of three-quark and quark-diquark components.

Additional information about the nucleon wave function may be obtained from the amplitudes corresponding
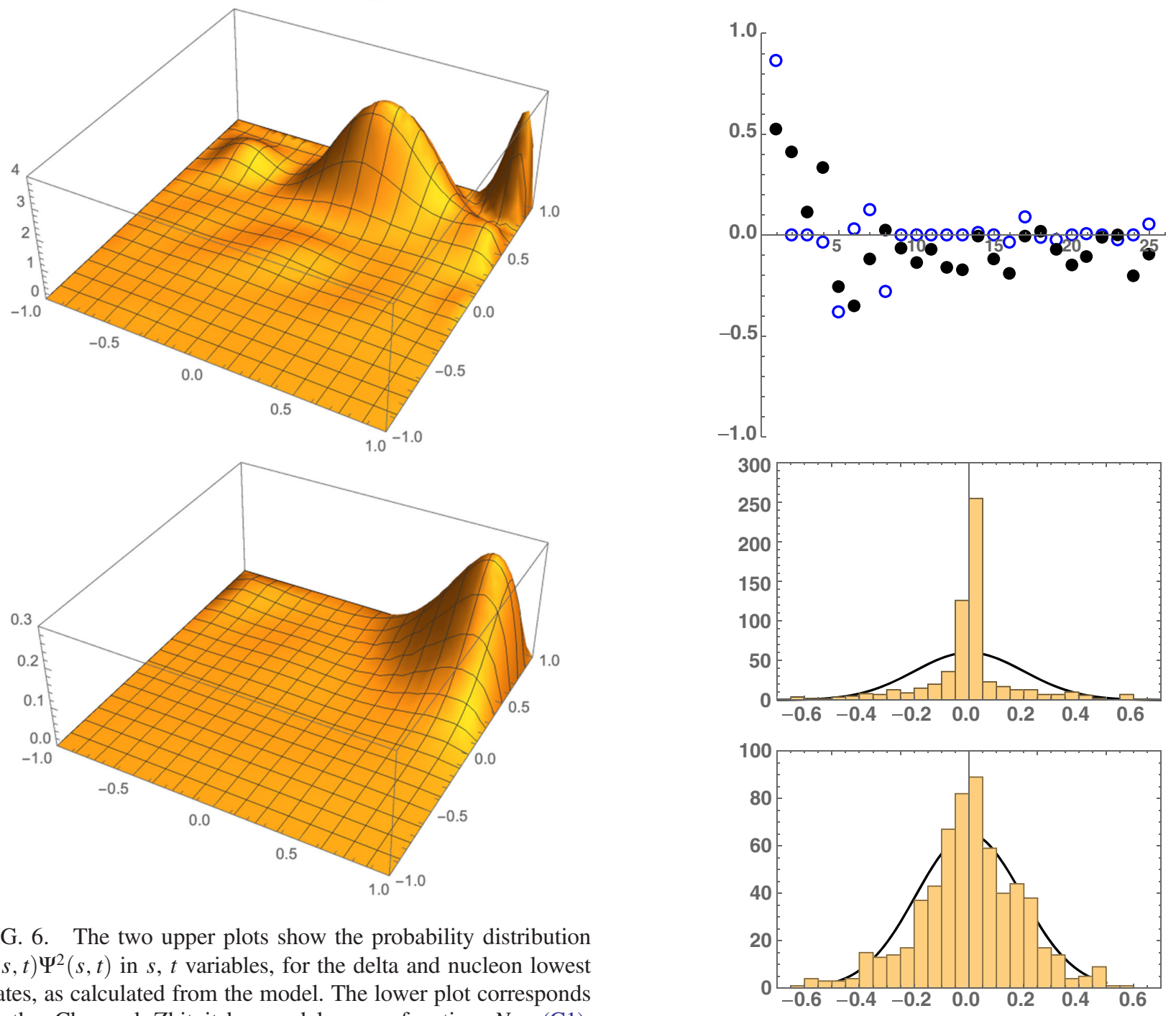

FIG. 6. The two upper plots show the probability distribution $J(s, t) \Psi^{2}(s, t)$ in $s, t$ variables, for the delta and nucleon lowest states, as calculated from the model. The lower plot corresponds to the Chernyak-Zhitnitsky model wave function $N_{I J}$ (C1), shown for comparison.

In order to reveal the structure, one can of course compare the wave functions without any integrations, as they depend on two variables $s, t$ only. Such plots, of $J(s, t) \Psi^{2}(s, t)$, we show in Fig. 6 for the delta, the nucleon, 
to particular basis states. We recall that its definition is in (10) [up to normalization, which we fixed from $\left.1=\int d s d t J(s, t) \psi_{n, l}(s, t)^{2}\right]$.

The wave function coefficients $C_{n}^{\alpha}$, defining expansion in the basis functions

$$
|\alpha\rangle=\sum_{n} C_{n}^{\alpha}|n\rangle
$$

are shown in Fig. 7. The upper plot compares those for the ground state delta and nucleon channels. Note first that the largest coefficients are the first [corresponding to trivial $\left.\psi_{0,0}(s, t) \sim \operatorname{const}(s, t)\right]$. Furthermore, for the delta it is close to 1 , while it is only $\sim 1 / 2$ for the nucleon. The fraction of "significant coefficients" is much larger for $N$. The nucleon wave function has a tail toward higher $n, l$ harmonics which does not show any decreasing trend. One may in fact conclude that convergence of the harmonic expansion is not there. This can be traced to behavior near $x=1$, perhaps the pointlike residual interaction leads to a true singularity there.

The two lower plots of Fig. 7 address the distribution of the wave function coefficients $C_{n}^{\alpha}$ in these two channels, without and with the residual four-quark interaction. It includes not only the ground state but the lowest 25 states in each channel. As is clear from these plots, in the former (delta) case the distribution is very non-Gaussian, with the majority of coefficients being small. The latter (nucleon) case, on the other hand, is in approximate agreement with Gaussian. In other quantum systems, e.g., atoms and nuclei, the Gaussian distribution of the wave function coefficients $C_{n}^{\alpha}$ is usually taken as a manifestation of "quantum chaos." In this language, we conclude that our model calculation shows that the residual four-quark interaction leads to the chaotic motion of quarks, at least inside the nucleon resonances. (If this conclusion surprises the reader, we remind them that the same interaction was shown to produce a chaotic quark condensate in vacuum. In particular, numerical studies of interacting instanton liquid in vacuum has led to the chiral random matrix theory of the vacuum Dirac eigenstates near zero, accurately confirmed by lattice studies.)

\section{PENTAQUARKS}

Jumping over the four-quark LFWFs we proceed to the next sector, with $q q q q \bar{q}$ composition. Although the main intention is to "unquench" our quark model, coupling sectors with different numbers of quarks together in the common Hamiltonian eigenstate, we start in this section with a study of the pentaquark $q q q q \bar{q}$ states as such. One can also view this section as a calculation of the Hamiltonian matrix in the $5 q$ sector, to be coupled with the $3 q$ one in the next section. For simplicity, we assume the four quarks to be in four distinguishable spin-flavor states, namely $u^{\uparrow} u^{\downarrow} d^{\uparrow} d^{\downarrow}$, which minimizes the Fermi repulsion effects by eliminating the exchange diagrams.

There is no change in the model or its parameters: all we do is write the Hamiltonian as a matrix in a certain basis and diagonalizing it. Four kinematical variables $z_{i}=s, t, u, w$, the integration measure $J(s, t, u, w)$ and the set of orthogonal functions were already defined in Sec. II. The presence of four integers, numbering the degree of the corresponding polynomials, naturally restricts our progress toward large values of each. Matrix elements of the Hamiltonian become in this sector four-dimensional integrals. Since this is a pilot study, restricted to laptop-based calculations inside Mathematica, we evaluate $\sim 10^{3}$ such matrix elements. The set of states $(l, m, n, k)$ used in this section contains 35 states for which $l+m+n+k<4$ listed in the Appendix D.

Matrix elements of the quark-mass term and the (longitudinal) confining terms, $H_{\text {mass }}=M_{q}^{2} \sum_{A=1,5}\left(1 / x_{A}\right)$ and $H_{\text {conf }}=-\left(\kappa^{4} / J / M_{q}^{2}\right)\left(\sum_{i=1,4} \partial_{z_{i}} J \partial_{z_{i}}\right)$, are calculated and diagonalized. Let us comment on some of the results.

The lowest eigenvalue was found to be $M_{\text {min.penta }}^{2}=$ $4.04 \mathrm{GeV}^{2}$. Following the procedure we adopted for mesons, we add the transverse motion term (treated as constant) $4 \times 0.12=0.48 \mathrm{GeV}^{2}$. Taking the square root, the predicted lowest mass of the light-flavor pentaquark (due to chiral mass and confinement) is therefore

$$
M_{\text {min .penta }}=2.13 \mathrm{GeV} .
$$

To get this number in perspective, let us briefly recall the history of the light pentaquark search. In 2003 the LEPS group reported a pentaquark $\Theta^{+}=u^{2} d^{2} \bar{s}$ with a surprisingly light mass, of only $1.54 \mathrm{GeV}, 0.6 \mathrm{GeV}$ lower than our calculation (and many others) yielded.

Of course, so far the residual perturbative and NJL-type forces were not included. Quick estimates of the time (including ours [20]) suggested that since the $u d$ diquark has a binding energy of $\Delta M_{u d} \approx-0.3 \mathrm{GeV}$ and the pentaquark candidate has two of them, one gets to the "then observed" mass of $1.54 \mathrm{GeV}$.

Several other experiments were also quick to report observations of this state, until other experiments (with better detectors and much higher statistics) showed that this pentaquark candidate does not really exist. A similar sad experimental status persists for all six-light-quark dibaryons, including the flavor symmetric $u^{2} d^{2} s^{2}$ spin-0 state much discussed in some theory papers.

A lesson for theorists is, as is often the case, not to trust any simple estimates but to proceed to some more consistent calculation. In the framework of an instanton liquid model, we studied diquark-diquark effective forces, by correlating pentaquark operators in a standard Euclidean setting [21]. We found that, like for baryons, consistently accounting for Fermi statistics generates strong repulsion between diquarks, basically canceling the presumed 


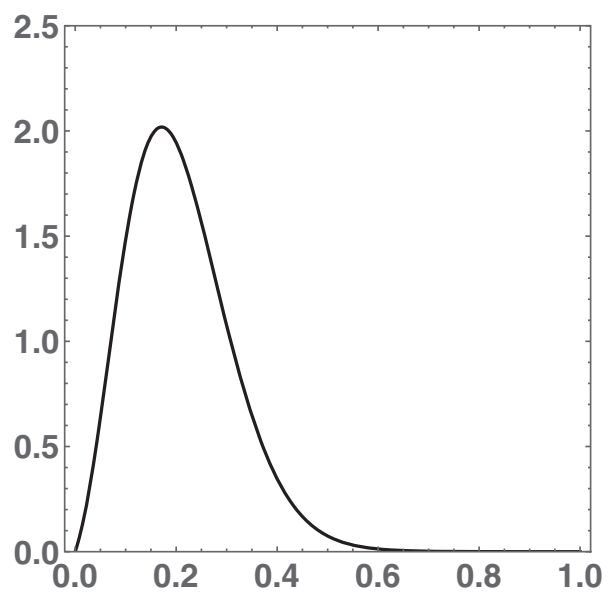

FIG. 8. Probability $P(X)$ to find a quark with momentum fraction $X$ in the lowest pentaquark state, calculated from the wave function given in the Appendix D. Note that in this WF the residual four-quark interaction has not yet been implemented.

attraction. The same trend was observed in multiple lattice studies, e.g., by the Budapest group [22]: no pentaquark states close to $1.54 \mathrm{GeV}$ in fact exist, while all physical states are much heavier.

Returning to our calculation, we have a few more comments. First, about the role of the confining term of the Hamiltonian: without it, the lowest eigenvalue is at $3.82 \mathrm{GeV}^{2}$, and with it is (as reported above) $4.04 \mathrm{GeV}^{2}$. The difference due to longitudinal confinement is thus $0.22 \mathrm{GeV}^{2}$. Twice this value (because there are two transverse directions) gives $0.44 \mathrm{GeV}^{2}$, very consistent with our empirical correction due to transverse motion of $4 \times 0.12=0.48 \mathrm{GeV}^{2}$.

The next-to-lowest eigenstate of the Hamiltonian has the mass $4.87 \mathrm{GeV}^{2}$, demonstrating a rather large gap with the lowest state. We have so far made no investigation of why it happens in this case, but not the others.

Even at the current level of approximation, without residual four-fermion interactions, the pentaquark wave function of the lightest eigenstate turns out to be rather nontrivial. Since it is a function of four variables, there is no simple way of plotting it. Suppressing a bunch of small terms, it can be written explicitly as a polynomial; see the Appendix D.

Note that the coefficients of highest order terms are not small, and in this sense the set of states was insufficient to show convergence. While there are nontrivial correlations between variables, a single-body distribution calculated from it does not exhibit anything unexpected; see Fig. 8.

\section{THE FIVE-QUARK SECTOR OF THE BARYONS}

As already mention in the Introduction, the initial motivation for this work was precisely a development of the framework in which one can consistently calculate the

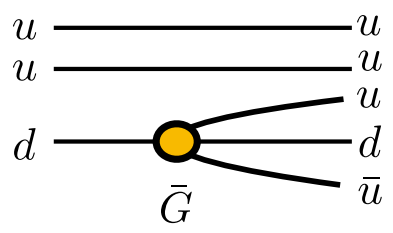

FIG. 9. The only diagram in which four-quark interaction connects the three- and five-quark sectors, generating the $\bar{u}$ sea.

wave functions in the lowest sector (three-quark for baryons), relating it to the observed parameters of the exclusive processes (form factors etc.) and the valence quark PDFs, and then proceed with the calculation of the other sectors of the physical state.

For the Hamiltonian matrix element corresponding to the diagram shown in Fig. 9 we calculated between the nucleon and each of the pentaquark wave functions, defined above, by the following $2+4$-dimensional integral over variables in the $3 q$ and $5 q$ sectors, related by certain delta functions:

$$
\begin{aligned}
& \langle N|H| 5 q, i\rangle=\bar{G} \int d s d t J(s, t) d s^{\prime} d t^{\prime} d u^{\prime} d w^{\prime} J\left(s^{\prime}, t^{\prime}, u^{\prime}, w^{\prime}\right) \\
& \psi_{N}(s, t) \delta\left(x_{1}-x_{1}^{\prime}\right) \delta\left(x_{2}-x_{2}^{\prime}\right) \psi_{i}\left(s^{\prime}, t^{\prime}, u^{\prime}, w^{\prime}\right) .
\end{aligned}
$$

The meaning of the delta functions is clear from the diagram; they are of course expressed via proper integration variables and numerically approximated by narrow Gaussians. After these matrix elements are calculated, the five-quark "tail" wave function is calculated via standard perturbation theory expression:

$\psi_{\text {tail }}\left(s^{\prime}, t^{\prime}, u^{\prime}, w^{\prime}\right)=-\sum_{i} \frac{\langle N|H| 5 q, i\rangle}{M_{i}^{2}-M_{N}^{2}} \psi_{i}\left(s^{\prime}, t^{\prime}, u^{\prime}, w^{\prime}\right)$.

The typical value of the overlap integral itself for different pentaquark states is $\sim 10^{-3}$, and using for effective coupling

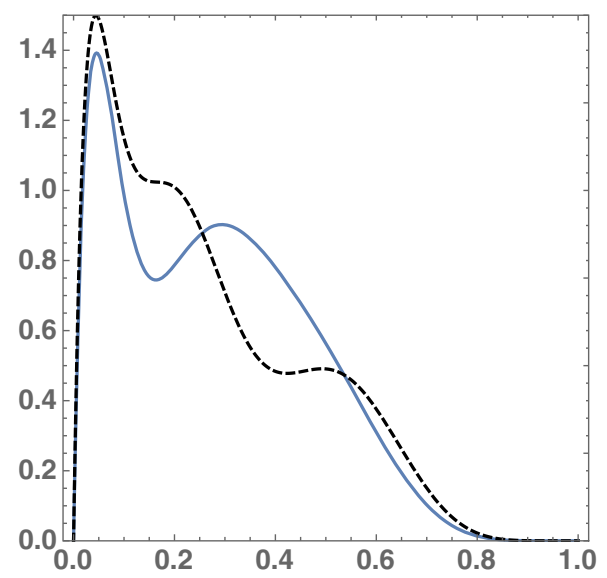

FIG. 10. The distribution over that $\bar{u}$ in its momentum fraction, for the nucleon and delta five-quark "tails" (solid and dashed lines, respectively). 
$\bar{G}$ the same value as we defined for $G$ from the nucleon, namely $\sim 17 \mathrm{GeV}^{2}$, one finds that the admixture of several pentaquarks to the nucleon is at the level of a percent. The resulting five-quark "tails" of the nucleon and delta baryons calculated in this way are given in the Appendix. The normalized distribution of the fifth body, namely $\bar{u}(x)$, over its momentum fraction is shown in Fig. 10. One can see a peak at $x_{\bar{u}} \sim 0.05$, which looks like a generic phenomenon. The oscillations at large $x_{\bar{u}}$ reflect strong correlations in the wave function between quarks, as well as perhaps indicate the insufficiently large functional basis used. This part of the distribution is perhaps numerically unreliable.

\section{PERTURBATIVE AND TOPOLOGY-INDUCED ANTIQUARK SEA}

The results of our calculation cannot be directly compared to the sea quark and antiquark PDFs, already plotted in Fig. 5, as those include large perturbative contributions, from gluon-induced quark pair production $g \rightarrow \bar{u} u, \bar{d} d$, dominant at very small $x$. However, these processes are basically flavor and chirality independent, while the observed flavor and spin asymmetries of the sea indicate that there must also exist some nonperturbative mechanism of its formation. For a general recent review see [16].

As originally emphasized by Dorokhov and Kochelev [23], the 't Hooft topology-induced four-quark interaction leads to processes

$$
u \rightarrow u(\bar{d} d), \quad d \rightarrow d(\bar{u} u)
$$

but not

$$
u \rightarrow u(\bar{u} u), \quad d \rightarrow d(\bar{d} d)
$$

which are forbidden by the Pauli principle applied to zero modes. Since there are two $u$ quarks and only one $d$ in the proton, one expects this mechanism to produce twice more $\bar{d}$ than $\bar{u}$.

The available experimental data, for the difference of the sea antiquark distributions $\bar{d}-\bar{u}$ (from [16]), are shown in Fig. 11. In this difference the symmetric gluon production should be canceled out, and therefore it is sensitive only to nonperturbative contributions.

A few comments are in order.

(i) First of all, the sign of the difference is indeed as predicted by the topological interaction: there are more anti-d than anti-u quarks.

(ii) Second, since $2-1=1$, this representation of the data directly gives us the nonperturbative antiquark production per valence quark, e.g., that of $\bar{u}$. This means that it can be directly compared to the distribution we calculated from the five-quark tail of the nucleon and delta baryons (Fig. 10).

(iii) The overall shape is qualitatively similar, although our calculation has a peak at $x_{\bar{u}} \sim 0.05$ while the

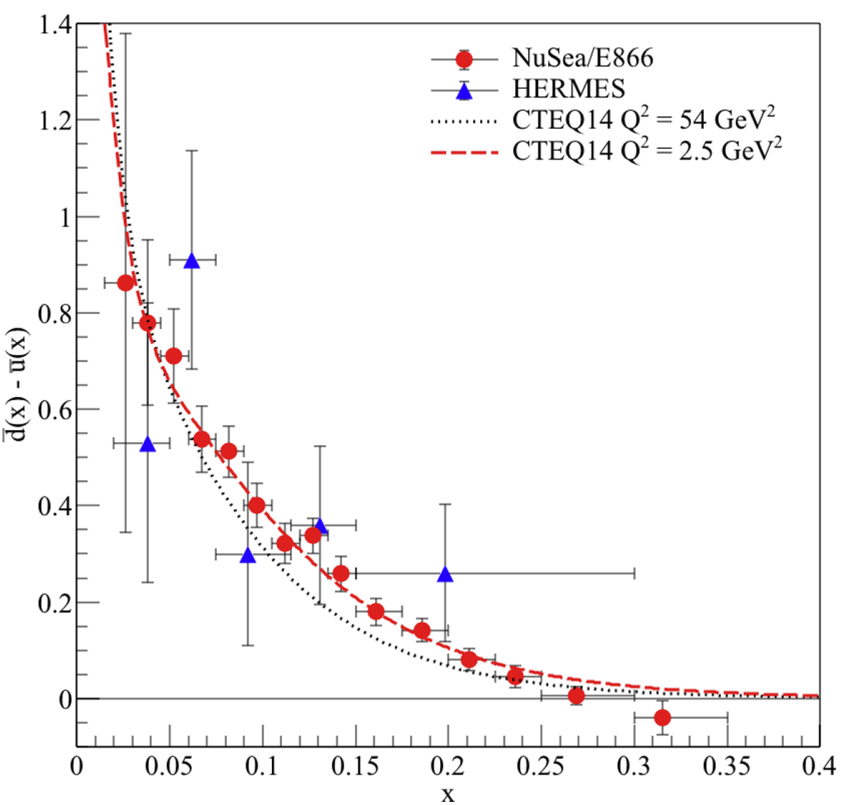

FIG. 11. The difference of sea antiquark momentum distributions, $\bar{d}-\bar{u}$, from Ref. [16].

experimental PDFs do not indicate it. Of course, there exist higher-quark-number sectors with seven or more quarks in baryons, which our calculation does not yet include; those should populate the small $x$ end of the PDFs.

(iv) The data indicate a much stronger decrease toward large $x_{\bar{u}}$ than the calculation.

(v) There are other theoretical models which also reproduce the flavor asymmetry of the sea, e.g., those with the pion cloud. In principle, one should be able to separate those and the topology-induced mechanism (that we focused on in this paper) by further combining flavor and spin asymmetry of the sea. In particular, as also noticed in [23], if a $d$ quark producing a $u \bar{u}$ pair has positive helicity, the sea quark and antiquark from 't Hooft four-quark operators must have the opposite (that is negative) helicity. The spin-zero pion mechanism, on the other hand, cannot transfer spin and would produce flavor but not spin sea asymmetry.

\section{SUMMARY AND DISCUSSION}

Application of some model Hamiltonians to light-front wave functions of hadrons should have been done a long time ago. Yet it is just starting, with the model presented in this paper still being (deliberately) rather schematic.

We follow the approach of Ref. [7], with the Hamiltonian including three terms: (i) the "constituent" light quark effective masses; (ii) the confinement term in harmonic form; and (iii) the local four-quark NJL-type interaction, here reduced to its 't Hooft version. The aim of this approach is to extend the model from meson to baryon 
three-quark states, then to five-quark states, and finally to the mixing between the two sectors.

For this pilot study we make a significant simplification of the model. We completely ignore transverse motion inside the hadrons, focusing on wave functions as a function of longitudinal momenta. We reduce the local four-fermion NJL-type effective interaction to that coming from gauge topology, believed to be the dominant part.

We have demonstrated that this simplified model does qualitatively describe the main features of meson and baryon mass splittings. The pion can become massless, while the $\eta^{\prime}$ is heavier than the $\rho$ meson. The nucleon is lighter than the $\Delta$ baryon, because of the attractive spinzero $u d$ diquark channel. All of this is already known for quite some time, from Euclidean approaches such as instanton liquid model and lattice QCD.

Our main findings are however not the hadronic masses, but the light-front wave functions. We have shown that local four-fermion residual interaction does indeed modify them in a very substantial way. In [7] it was shown that the $\pi$ and $\rho$ meson have very different wave functions. We now show that it is also true for baryons: the proton and the $\Delta$ have qualitatively different wave functions. The imprint left by strong diquark $(u d)$ correlations on the light-front wave functions is now established. We furthermore found evidence that nucleon resonances show features of "quantum chaos" in quark motion.

We also made the first steps toward the "unquenching" of the light-front wave functions, estimating multiple matrix elements of the mixings between 3-q and 5-q (baryonpentaquark) components of the nucleon wave function. This puts mysterious spin and flavor asymmetries of the nucleon sea inside the domain of consistent Hamiltonian calculation.

Now, moving on to the discussion part, let us point out several directions in which the model itself, as well as the calculations presented, can be improved.

As technical issues are concerned, the most direct (but important) generalization of this work would be to repeat these calculations in a significantly larger function space. In particular, one needs to restore the dependence of the wave function on transverse motion and on explicit spin states of the quarks.

It would be just a straightforward exercise to generalize this work to strange mesons and baryons. We already discussed (around Fig. 1) that $(\bar{u} u)(\bar{d} d)$ interaction includes comparable contributions from diagrams (b) and (c), while $(\bar{s} s)(\bar{d} d),(\bar{u} u)(\bar{s} s)$ only have the diagram (d): light quark masses are too small to include sizable analogs of the diagram (b). Therefore $S U(3)_{f}$ breaking is expected to be substantial.

As for the model, in its current form we only get a schematic understanding of multiquark correlations in the LFWFs. One can use instead more realistic NJL-type interactions, as used in modern models, including mean value of the Polyakov line as a dynamical variable, or in works including its functional renormalization group flow, e.g., [24]. Yet, the main thing which needs improvement is to complement the current local form of the four-fermion interaction by a form with certain realistic form factors. Even the original NJL model had a cutoff parameter $\Lambda$, above which the interaction no longer exists. In the instanton liquid version, the form factors have the form $\sim \exp (-p \rho)$ where $\rho \sim 1 / 600 \mathrm{MeV}$ is the instanton size. The current model uses a purely local form, assuming that topological solitons are small compared to hadronic sizes. Yet one needs these form factors to be perhaps able to finally find good convergence in the functional space of harmonics.

Needless to say, many applications of the LFWFs we did not even touch. For example, one can calculate cross sections of various exclusive processes, e.g., electromagnetic form factors or large angle scattering. Perhaps one should only do so when the model used becomes more realistic and quantitative.

Finally, we comment on our general goal of bridging the nonperturbative models with observed PDFs and perturbative evolution. The pQCD evolution of the baryon wave functions can be included if needed, as the (matrix of) anomalous dimensions has been calculated quite some time ago [15]. The multibody wave functions, if known, allow us to do more than just predict the PDFs: one may in particular evaluate the matrix elements of the higher-twist operators. So far, the only effort in this direction we are aware of is Ref. [25] in which four-body $q^{3} g$ wave functions were derived and used for twist-3 estimates. Comparing their properties with experiment at medium $Q^{2}$ would eventually provide a satisfactory "bridge" between the perturbative and nonperturbative views on the hadronic structure.

\section{ACKNOWLEDGMENTS}

The author thanks Felix Israilev for very useful discussion of quantum chaos in many-body systems. The work is supported by the U.S. Department of Energy, Office of Science, under Contract No. DE-FG-88ER40388.

\section{APPENDIX A: THE TWO-BODY LFWFs FOR PION, RHO AND ETA' MESONS}

The approximate form of three mesonic wave functions are given below. The measure times their squares is plotted in Fig. 2. The only comment is about convergence of the series: the first one, for $\rho$, clearly shows sign of convergence, as the maximal coefficient is at the middle term. The pion seems to have a singularity at the end points, while eta' has zero. Here we give approximate forms of three mesonic wave functions. (Note that for mesons $z$ simply means the variable $s$ in Eq. (4).) 


$$
\begin{aligned}
\psi_{\rho}(s) \approx & -1.56263+13.5307 z^{2}-55.002 z^{4} \\
& +138.857 z^{6}-241.184 z^{8}+298.688 z^{10}-261.438 z^{12} \\
& +153.563 z^{14}-53.9465 z^{16}+8.49524 z^{18} \\
\psi_{\pi}(s) \approx & -0.727959-3.13128 z^{2}+66.7135 z^{4} \\
& -681.778 z^{6}+3689.13 z^{8}-11582.8 z^{10}+21693.6 z^{12} \\
& -23823.4 z^{14}+14124.3 z^{16}-3485.37 z^{18} \\
\psi_{\eta^{\prime}}(s) \approx & -1.77724+26.6155 z^{2}-147.594 z^{4} \\
& +518.514 z^{6}-1365.06 z^{8}+2750.13 z^{10}-3971.84 z^{12} \\
& +3738.27 z^{14}-2012.73 z^{16}+465.809 z^{18} .
\end{aligned}
$$

\section{APPENDIX B: THE THREE-BODY LFWFS FOR NUCLEON AND DELTA BARYONS}

The 25 lowest harmonics shown in Fig. 7 correspond to the following set of the $n, l$ values:

$(0,0),(0,1),(1,1),(1,0),(0,2),(1,2),(2,2),(2,0)$,

$(2,1),(0,3),(1,3),(2,3),(3,3),(3,2),(3,1),(3,0)$,

$(0,4),(1,4),(2,4),(3,4),(4,4),(4,3),(4,2),(4,1),(4,0)$.

The functional set is defined in (24).

The approximate polynomial form of the light-front wave functions of the nucleon and delta baryons, in $s, t$ variables, is

$$
\begin{aligned}
\psi_{N} \sim & -2.34+11.7 t+43.5 t^{2}-4.78 t^{3}-48.7 t^{4} \\
& +s^{4}\left(-26.2+16.8 t+316 . t^{2}+14.2 t^{3}-523 . t^{4}\right) \\
& +s\left(7.98-29.3 t+31.4 t^{2}+115 . t^{3}-97.3 t^{4}\right) \\
& +s^{3}\left(-12.4+57.4 t+45 . t^{2}-169 . t^{3}-30 . t^{4}\right) \\
& +s^{2}\left(22.3-32 . t-239 . t^{2}-11.2 t^{3}+347 . t^{4}\right) \\
\psi_{\Delta} \approx & -12.7-23.1 t+39.9 t^{2}+36.2 t^{3}-39.5 t^{4} \\
& +s^{2}\left(39.2+69.9 t-121 . t^{2}-109 . t^{3}+120 . t^{4}\right) \\
& +s^{4}\left(-28.1-49.4 t+86.9 t^{2}+78.0 t^{3}-86.3 t^{4}\right) .
\end{aligned}
$$

\section{APPENDIX C: MODELS FOR NUCLEON LFWF USED BY CHERNYAK, OGLOBLIN AND ZHITNITSKY}

In the paper [19] one finds usage of at least three different nucleon LFWFs, which came from a different usage of the QCD sum rules method in the 1980s. For definiteness, we reproduce it here:

$$
\begin{aligned}
N_{I J}= & 23.814 x_{1}^{2}+12.978 x_{2}^{2}+6.174 x_{3}^{2}+5.88 x_{3}-7.098 \\
N_{K S}= & 20.16 x_{1}^{2}+15.12 x_{2}^{2}+22.68 x_{3}^{2}-6.72 x_{3} \\
& +1.68\left(x_{1}-x_{2}\right)-5.04 \\
N_{J}= & 18.06 x_{1}^{2}+4.62 x_{2}^{2}+8.82 x_{3}^{2}-1.68 x_{3}-2.94 . \quad(\mathrm{C} 1)
\end{aligned}
$$

However, for the purpose of comparison we used only one of them, $N_{I J}$, in Fig. 6, since they are all qualitatively similar.

\section{APPENDIX D: THE FIVE-BODY LFWF OF THE LOWEST PENTAQUARK}

For definiteness, we indicate the specific set of five-body wave functions used. In the notations of (17), the set of states $(l, m, n, k)$ used in this section contains the following states for which $l+m+n+k<4,35$ in total:

$(0,0,0,0),(1,0,0,0),(0,1,0,0),(0,0,1,0),(0,0,0,1)$,

$(1,1,0,0),(1,0,1,0),(1,0,0,1),(0,1,1,0),(0,1,0,1)$,

$(0,0,1,1),(2,0,0,0),(0,2,0,0),(0,0,2,0),(0,0,0,2)$,

$(0,1,1,1),(1,0,1,1),(1,1,0,1),(1,1,1,0),(2,1,0,0)$,

$(2,0,1,0),(2,0,0,1),(1,2,0,0),(0,2,1,0),(0,2,0,1)$,

$(1,0,2,0),(0,1,2,0),(0,0,2,1),(1,0,0,2),(0,1,0,2)$,

$(0,0,1,2),(3,0,0,0),(0,3,0,0),(0,0,3,0),(0,0,0,3)$.

The quark mass and confinement terms of the Hamiltonian, after diagonalization, produced the following mass spectrum (in $\mathrm{GeV}^{2}$ ):

$M_{5 q}^{2} \approx 13.9,13.7,12.4,12.2,11.0,10.9,10.9,10.8,10.6$,

$10.6,10.4,10.3,10.3,10.3,10.3,10.1,10.0,9.97$,

$9.90,9.76,8.60,8.20,8.14,8.13,8.04,8.02,7.89$,

7.76, 7.71, 7.64, 5.16, 5.05, 4.94, 4.84, 4.04.

The WF of them we will not present except for the lowest (the last). Its approximate form is

$$
\begin{aligned}
\psi_{\text {penta }}= & 1 .-1.21 t^{3}+1.61 u-0.519 u^{2}-2.03 u^{3} \\
& +s^{2}(-1.79+0.0622 t+0.177 u+0.248 w) \\
& +t^{2}(-1.40+0.265 u+0.341 w)+1.46 w \\
& -0.488 u w+0.510 u^{2} w+0.835 w^{2}+0.194 u w^{2} \\
& -2.81 w^{3}+t\left(1.19-0.117 u+0.059 u^{2}-0.18 w\right. \\
& \left.-0.018 u w+0.074 w^{2}\right)
\end{aligned}
$$

where harmonics with smaller coefficients are neglected. 
APPENDIX E: THE LFWF IN THE FIVE-QUARK SECTOR OF THE NUCLEON AND DELTA BARYONS

The five-quark "tails" of the nucleon and the delta baryons that we calculated have (in arbitrary normalization) the following wave functions:

$$
\begin{aligned}
\psi_{N}^{5 q} \approx & -0.88-1.08 s^{3}+2.89 t^{3}+3.55 u-4.66 u^{2}+t^{2}(2.46-4.65 w) \\
& +s^{2}(-0.44-0.42 t+3.9 u-1.83 w)-0.40 w-3.3 u w+9.2 u^{2} w \\
& +4.45 w^{2}-7.4 u w^{2}-1 . w^{3}+t\left(0.94-4.32 u-2.68 u^{2}-3.12 w\right. \\
& +10.1 u w)+s\left(1.86+1.96 t^{2}-1.914 u+t(0.25-2.48 u)-7.0 w+4.58 u w+5.36 w^{2}\right)
\end{aligned}
$$

and

$$
\begin{aligned}
\psi_{\Delta}^{5 q} \approx & 1.78-2.80 s^{3}+7.49 t^{3}-6.54 u+3.33 u^{2}+s^{2}(3.50-1.09 t+0.91 u-4.73 w)-8.25 w+20.1 u w \\
& -1.71 u^{2} w+11.5 w^{2}-19.2 u w^{2}-2.57 w^{3}+t^{2}(-5.35+1.19 w)+t\left(-1.09+4.66 u-6.91 u^{2}-0.96 w+1.44 u w\right) \\
& +s\left(0.05-0.20 t+0.40 t^{2}-0.21 u+2.61 w+0.35 u w-1.08 w^{2}\right) .
\end{aligned}
$$

[1] E. V. Shuryak, Rev. Mod. Phys. 65, 1 (1993).

[2] S. J. Brodsky and G. P. Lepage, Adv. Ser. Dir. High Energy Phys. 5, 93 (1989).

[3] V. L. Chernyak and A. R. Zhitnitsky, Phys. Rep. 112, 173 (1984).

[4] J. Erlich, E. Katz, D. T. Son, and M. A. Stephanov, Phys. Rev. Lett. 95, 261602 (2005).

[5] M. Jarvinen and E. Kiritsis, J. High Energy Phys. 03 (2012) 002.

[6] S. J. Brodsky and G. F. de Teramond, Phys. Rev. Lett. 96, 201601 (2006).

[7] S. Jia and J. P. Vary, Phys. Rev. C 99, 035206 (2019).

[8] G. 't Hooft, Phys. Rev. D 14, 3432 (1976); 18, 2199(E) (1978).

[9] Y. Nambu and G. Jona-Lasinio, Phys. Rev. 122, 345 (1961).

[10] E. V. Shuryak, Nucl. Phys. B203, 93 (1982).

[11] T. Schafer and E. V. Shuryak, Rev. Mod. Phys. 70, 323 (1998).

[12] G. S. Bali et al. (RQCD Collaboration), Eur. Phys. J. A 55, 116 (2019).

[13] D. E. Kharzeev and E. M. Levin, Phys. Rev. D 95, 114008 (2017).
[14] E. V. Shuryak and A. I. Vainshtein, Nucl. Phys. B199, 451 (1982).

[15] V. M. Braun, S. E. Derkachov, G. P. Korchemsky, and A. N. Manashov, Nucl. Phys. B553, 355 (1999).

[16] D. F. Geesaman and P. E. Reimer, Rep. Prog. Phys. 82, 046301 (2019).

[17] V. Y. Petrov, M. V. Polyakov, R. Ruskov, C. Weiss, and K. Goeke, Phys. Rev. D 59, 114018 (1999).

[18] V. M. Braun, Continuous Advances in QCD 2006 (World Scientific, Singapore, 2007), pp. 42-57.

[19] V.L. Chernyak, A. A. Ogloblin, and I. R. Zhitnitsky, Z. Phys. C 42, 583 (1989); [Yad. Fiz. 48, 1398 (1988)] [Sov. J. Nucl. Phys. 48, 889 (1988)].

[20] E. Shuryak and I. Zahed, Phys. Lett. B 589, 21 (2004).

[21] E. V. Shuryak, J. Phys. Conf. Ser. 9, 213 (2005).

[22] F. Csikor, Z. Fodor, S. D. Katz, T. G. Kovacs, and B. C. Toth, Nucl. Phys. B, Proc. Suppl. 153, 49 (2006).

[23] A. E. Dorokhov and N. I. Kochelev, Phys. Lett. B 304, 167 (1993).

[24] M. Drews and W. Weise, Prog. Part. Nucl. Phys. 93, 69 (2017).

[25] V. M. Braun, T. Lautenschlager, A. N. Manashov, and B. Pirnay, Phys. Rev. D 83, 094023 (2011). 